\title{
Utilité du radar Doppler dans l'étude des précipitations
}

\author{
The rolle of Doppler radar \\ in the study of precipitation
}

\author{
P. Amayenc \\ Centre de recherches en Physique de l'environnement \\ CNET/CNRS, Issy-les-Moulineaux
}

\section{Introduction}

Les radars conventionnels destinés à l'observation des précipitations effectuent une mesure de réflectivité à partir de la puissance des échos reçus par rétrodiffusion sur les hydrométéores contenus dans chaque volume diffusant exploré. La notion de déplacement des cibles n'intervient pas. En réalité, les hydrométéores sont animés de mouvements qui résultent de l'entraînement (supposé parfait) de l'air, et de leur vitesse de chute gravitationnelle.

Le signal renvoyé par chaque cible est affecté d'un décalage Doppler en fréquence

$$
\Delta f=-2 V_{R} / \lambda
$$

où $V_{R}$ est la projection de la vitesse de la cible sur la direction de visée

et $\lambda$, la longueur d'onde de travail.

La puissance reçue est répartie suivant un spectre $S(f) d f$ (de forme généralement proche d'une gaussienne) que lon peut traduire en spectre de vitesse Doppler $S(V) d V$ dans chaque intervalle de vitesse $[V, V+d V]$, associé à l'intervalle de fréquence $[f, f+d f]$.

Le principe du radar Doppler est de permettre la mesure de ce spectre ou, du moins, de ses caractéristiques qui sont sa puissance (intégrale), sa vitesse moyenne (position) et son écart-type (largeur), quantités que l'on peut calculer à partir des trois premiers moments normalisés du spectre.

La puissance du spectre conduit à la définition classique du facteur de réflectivité radar $Z$. Pour un radar opérant avec un faisceau incliné d'un angle de site $\alpha$ sur l'horizontale, la vitesse moyenne Doppler $V_{R}$ est donnée par:

$$
V_{R}=U_{R}+V_{T} \sin \alpha
$$

où $U_{R}$ est la vitesse radiale moyenne de l'air dans le volume diffusant considéré et $V_{T} \sin \alpha$ représente la projection sur la direction de visée, de la vitesse de chute moyenne $V_{T}$ des hydrométéores; l'importance relative de ces deux contributions à la mesure de $V_{R}$ dépend de l'angle de visée $\alpha$ du radar.

L'écart-type $\sigma$ des vitesses Doppler peut être décomposé en plusieurs contributions généralement indépendantes qui résultent pour l'essentiel du gradient de la vitesse radiale de l'air dans le volume diffusant, de l'effet des mouvements turbulents d'échelle inférieure aux dimensions de ce volume et de la dispersion des vitesses de chute des cibles. Moyennant des procédures d'utilisation appropriées d'un ou de plusieurs radars Doppler, il est donc possible d'obtenir des informations sur la dynamique des particules précipitantes, interprétables en termes de dynamique de l'air et/ou de vitesse de chute, dans chaque porte de distance au sein des systèmes précipitants observés.

Le présent article n'a pas un caractère exhaustif dans l'exposé des méthodologies d'utilisation des radars Doppler pour étudier les précipitations. Nous nous sommes limités, dans les sections 2 et 3 à la présentation des trois techniques les plus employées par les expérimentateurs, en nous attachant à exposer leurs fondements et à indiquer quelques résultats expérimentaux typiques qu'elles permettent d'obtenir. Pour compléter cette présentation, nécessairement sélective, le lecteur intéressé pourra consulter par exemple Battan [1] ou Sauvageot [2] et les bibliographies associées. La dernière section évoque brièvement le contexte actuel d'utilisation des radars Doppler destinés à l'étude des précipitations et les développements prévisibles.

\section{Le tir vertical: vitesse verticale de l'air et distribution granulométrique des précipi- tations.}

Dans chaque volume diffusant exploré en visée verticale par un radar Doppler, la vitesse radiale moyenne $V$

LA HOUILLE BLANCHE/N ${ }^{\circ}$ 5/6-1983 
mesurée représente, d'après l'équation (1), la somme algébrique du mouvement vertical moyen $w$ de l'air et de la vitesse de chute gravitionnelle moyenne $V_{T}$ des hydrométéores :

$$
V=w+V_{T}
$$

On voit que, si l'une des deux quantités $w$ ou $V_{T}$ est connue, la mesure de $V$ permet de déduire l'autre. Si l'on se place dans le cas de la diffusion de Rayleigh, sur des cibles sphériques de même nature et si l'on peut négliger l'élargissement spectral dû à la turbulence de l'air (ceci nécessite en particulier une faible ouverture du faisceau radar, de l'ordre de $1^{\circ}$ ), le spectre Doppler peut être interprété comme le spectre des vitesses de chute des précipitations:

$$
S\left(y_{T}\right) d v_{T}=n(D) D^{6} d D
$$

$S\left(v_{T}\right) d v_{T}$ est le facteur de réflectivité des particules de diamètre compris entre $D$ et $D+d D$, dont la distribution dimensionnelle par unité de volume est représentée par $n(D)$, et qui chutent à des vitesses comprises entre $v_{T}$ et $v_{T}+d v_{T}$. La vitesse verticale moyenne de l'air n'étant généralement pas nulle, l'écriture de l'équation (3) suppose que l'on a effectué une translation du spectre Doppler $S(V) d V$ suivant l'axe des vitesses, d'une quantité égale à $w$, de façon à l'amener à la position qu'il occuperait pour $w=0$. Si l'on sait en outre relier la vitesse de chute $v_{T}$ d'une particule précipitante à son diamètre $D$ par une loi $v_{T}(D)$, adaptée le cas échéant à la nature de la précipitation et corrigée de l'effet de variation de la densité atmosphérique avec l'altitude, on peut obtenir la distribution granulométrique des précipitations par l'équation :

$$
n(D)=D^{-6} S\left(v_{T}\right) d v_{T} / d D
$$

La précision obtenue sur $n(D)$ dépend fortement de celle avec laquelle est calculée $w$. Inversement, on montre que l'utilisation d'hypothèses raisonnables sur la distribution $n(D)$ permet de déterminer la vitesse moyenne de chute $V_{T}$; la visée verticale permet alors de mesurer la vitesse verticale $w$ de l'air. Il est ensuite possible de revenir à l'équation (4) pour calculer la distribution $n(D)$. Notons que, dans le cas de la glace, on montre que les considérations précédentes s'appliquent, à condition de considérer que le diamètre qui intervient est celui qu'aurait la goutte d'eau sphérique résultant de la fonte du cristal.

Trois méthodes principales ont été utilisées pour déterminer $w$ et $n(D)$. La plus élémentaire consiste à se donner la vitesse de chute en air calme des plus petites particules détectables par le radar utilisé ( $D \sim 150 \mu \mathrm{m}$ pour les radars opérant dans la gamme de longueurs d'onde $3-10 \mathrm{~cm}$ ). Ceci permet de placer directement un bord du spectre sur l'échelle des vitesses de chute (Battan [3]; Battan et Theiss [4]). Bien adaptée au cas de la neige, cette méthode souffre d'un manque de précision qui la rend à peu près inexploitable dans tous les autres cas. Une autre méthode consiste à utiliser une relation $V_{T}-Z$. Une telle relation peut être établie à partir de considérations théoriques sur la forme de la distribution $n(D)$, supposée par exemple exponentielle selon la loi bien connue de Marshall et Palmer [5], dans le cas de la pluie:

$$
n(D)=N_{0} \exp (-\Lambda D) \text { avec } N_{0}=0.08 \mathrm{~cm}^{-4}
$$

Cette méthode, proposée à l'origine par Rogers [6], foumit la relation :

$$
V_{T}=3.8 Z^{1 / 14}\left(V_{T} \text { en } \mathrm{ms}^{-1}, Z \text { en } \mathrm{mm}^{6} \mathrm{~m}^{-3}\right)
$$

utilisable pour la pluie, au niveau du sol. Il est possible d'obtenir des relations analogues pour d'autres types de précipitations (neige, cristaux, grêle), éventuellement à partir de mesures de distributions au sol ou à bord d'un avion instrumenté. Enfin, pour éviter d'obtenir parfois des résultats de distributions $n(D)$ dont la forme diffère sensiblement de celle supposée à priori, une troisième approche, moins restrictive, consiste à réaliser un ajustement entre le spectre Doppler expérimental et un spectre théorique dépendant des trois paramètres $N_{0}$, $\Lambda$ (caractérisant $n(D))$ et $w$ qui peuvent ainsi être déterminés simultanément (Hauser et Amayenc [7]).

Partant de ces considérations, de nombreux travaux ont été réalisés depuis vingt ans pour exploiter les observations d'un radar Doppler en tir vertical au sein de précipitations de caractère stratiforme ou orageux (voir par exemple Atlas et al. [8]). La connaissance de la vitesse verticale est une composante essentielle de la dynamique des systèmes précipitants, quant aux distributions dimensionnelles des hydrométéores, leur connaissance en fonction de l'altitude traduit les mécanismes de transformation (croissance, évaporation, etc...) et d'évolution des précipitations. Ce type d'observation utilise évidemment le passage du système précipitant au-dessus du radar qui opère alors une exploration altitude-temps dans un volume imposé par le déplacement du système.

L'exemple de résultats que nous présentons cidessous, a été obtenu avec le système Ronsard (Nutten et al. [9]) de deux radars météorologiques Doppler du C.R.P.E. Il est relatif à un orage observé dans la région de Zurich par le radar $R_{1}$ opérant en tir vertical. Le deuxième radar $R_{2}$, situé à environ $30 \mathrm{~km}$ de $R_{1}$, réalisait simultanément une surveillance en volume de l'orage (se déplaçant horizontalement à la vitesse d'environ $20 \mathrm{~ms}^{-1}$ ) afin de situer les observations du radar $R_{1}$ au sein de l'évènement. Le traitement des données du radar $R_{1}$ utilisait une relation empirique $V_{T}-Z$, adaptée aux observations, pour déduire $w$ et $n(D)$ en fonction de l'altitude selon une procédure évoquée auparavant (Hauser et Amayenc [10]).

Un résultat du traitement des données acquises au cours d'une séquence de $135 \mathrm{~s}$ (équivalente à une distance horizontale de $2,7 \mathrm{~km}$ en raison du déplacement du système) est reportée sur la figure 1 . Celle-ci représente, en fonction de l'altitude, les moyennes de divers paramètres déduits de l'analyse avec mention pour chacun d'eux, de l'écart-type dû à sa variation temporelle au cours de l'acquisition. On note la présence d'ascendances importantes au sein de la cellule orageuse observée ( $w$ atteint environ $10 \mathrm{~ms}^{-1}$ ) ainsi que des profils de $Z, V, M, D_{0}, \Lambda$ qui traduisent des variations significatives de la structure verticale des caractéristiques des précipitations.

La traversée de l'isotherme $0^{\circ} \mathrm{C}$ (situé vers $3,2 \mathrm{~km}$ ) 
GROSSDIETWIL $\quad$ 18-7-78 1646 TU
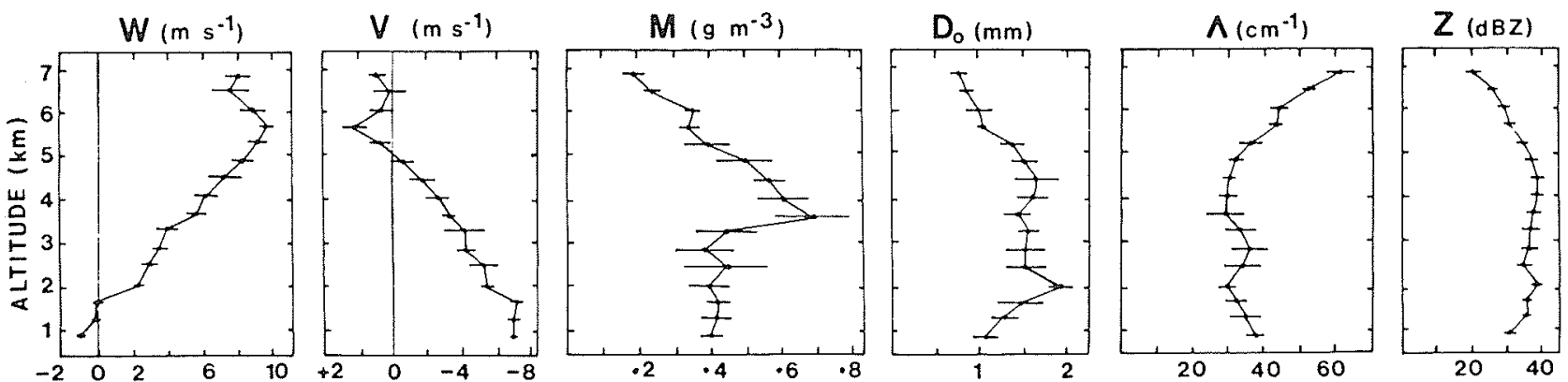

Figure 1 - Profils verticaux de paramètres caractéristiques des précipitations déduits de l'observation d'une cellule orageuse à Grossdietwil (Suisse) par un radar Doppler en tir vertical, le 18 juillet 1978: V (vitesse Doppler), Z Facteur de réflectivité radar), M (teneur en eau précipitante), $D_{0}$ (diamètre médian de la distribution dimensionnelle des gouttes), $\Lambda$ (pente de la distribution), $w$ (vitesse verticale de l'air). Les vitesses w et $V$ sont positives vers le haut. D'après Hauser et Amayenc [10].

n'est pas accompagnée ici de phénomène de bande brillante "radar" en raison du brassage convectif important au sein de l'orage. D'autres éléments de l'analyse confirment la présence d'eau liquide au moins jusqu'à $5,5 \mathrm{~km}$ d'altitude. Ce niveau correspond à peu près à une région où les hydrométéores sont en suspension $(V=0)$ sous les effets compensatoires de l'ascendance de l'air et de la vitesse de chute gravitationnelle. Les profils verticaux de $D_{0}$ et $\Lambda$ caractérisent des distributions de gouttes dont les dimensions croissent du haut vers le bas avec une brusque décroissance endessous de $2 \mathrm{~km}$.

Ces résultats illustrent à la fois l'intérêt de l'analyse et l'une de ses limites, qui tient au fait que les variations observées en fonction de l'altitude, ne traduisent pas nécessairement l'évolution des caractéristiques d'une précipitation "en suivant sa chute". Dans l'exemple présenté, on peut en effet montrer que les trajectoires des précipitations traversent le plan d'observation altitude-temps du radar en visée verticale. L'intérêt du deuxième radar réalisant une surveillance à plus grande échelle est ici évident.

Un autre résultat obtenu dans cette étude est reporté sur la figure 2 . On a représenté les relations obtenues entre le facteur de réflectivité et le taux précipitant calculés à partir des résultats de distributions des gouttes de pluie dans la région située entre le sol et $3,2 \mathrm{~km}$ d'altitude. Dans la figure $2 . a$, il s'agit du taux précipitant $R$ mettant en jeu la vitesse de chute intrinsèque des précipitations (déduction faite de la vitesse verticale de l'air, déterminée simultanément). Le taux précipitant $R^{*}$ (Fig. 2.b) est le taux effectif mettant en jeu la vitesse de chute nette (modifiée par la vitesse verticale de l'air). On note que la relation $Z-R *$ est nettement dégradée par rapport à la relation $Z-R$, en raison des effets de dispersion introduits par la présence de mouvements verticaux importants (dans l'échantillon utilisé, les valeurs de $w$ sont comprises entre -2 et $+6 \mathrm{~m} \mathrm{~s}^{-1}$ ). Ce résultat traduit directement une cause d'erreur irréductible dans les mesures hydrologiques par radar, dans le cas de la convection orageuse.

\section{Dynamique des systèmes précipitants.}

Lorsque le radar est dirigé dans une direction d'azimut $\beta$ (par rapport au Nord suivant un axe $y$ ) et d'élé- vation $\alpha$, la vitesse radiale $V_{r}$ mesurée à la distance :

$$
r=\left(x^{2}+y^{2}+z^{2}\right)^{1 / 2}
$$

est donnée, d'après l'équation (1), par :

$$
V_{r}=u \cos \alpha \sin \beta+v \cos \alpha \cos \beta+V_{z} \sin \alpha
$$
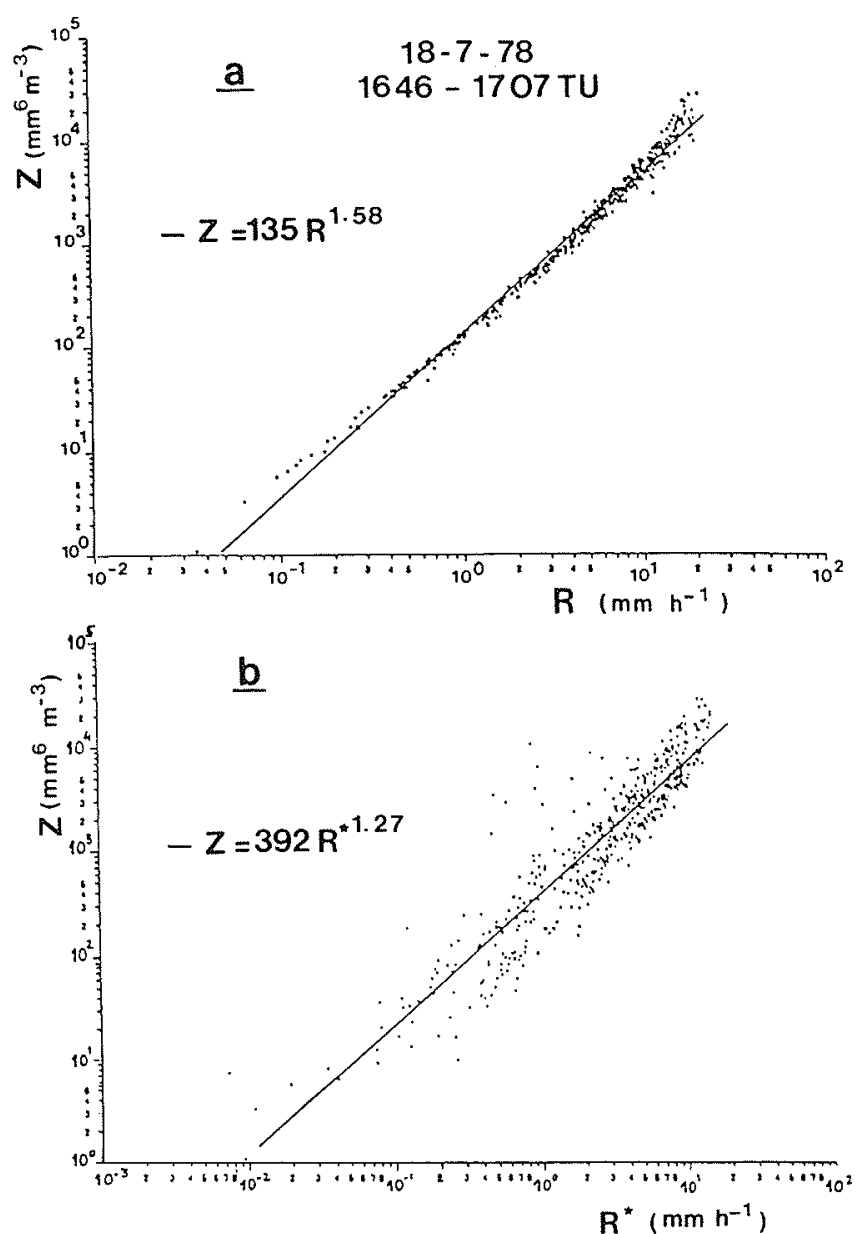

Figure 2 - Relations entre le factelir de réflectivité radar $Z$ et le taux précipitant $R$ que l'on aurait en air calme (Fig. 4.a) ou le taux précipitant net $R^{*}$, incluant l'effet des mouvements verticaux de l'air (Fig. 4.b). Les résultats sont établis à partir des données de radar Doppler en tir vertical entre le sol et $3.2 \mathrm{~km}$ d'altitude (463 points), dans l'orage mentionné à propos de la figure 1. 
où $V_{z}=w+V_{T}$ et $u, v, w$ sont les composantes de la vitesse de l'air suivant les axes $x, y, z$, d'un trièdre trirectangle $(O, x, y, z)$ d'origine située au radar, l'axe $z$ étant dirigé suivant la verticale. La possibilité de déterminer les composantes du mouvement de l'air à partir des mesures de vitesse radiale par un ou plusieurs radars Doppler a été largement exploitée. Nous décrivons ci-dessous les deux méthodologies les plus utilisées.

\subsection{Méthodologie V.A.D. : Ecoulement de l'air à moyenne échelle.}

Lhermitte et Atlas [11] ont proposé une procédure d'utilisation d'un radar Doppler pour mesurer les profils verticaux de vent au sein d'un système précipitant étendu, homogène et uniforme à chaque altitude. Cette technique, dénommée VAD (pour Velocity Azimuth Display), consiste à effectuer des rotations de l'antenne à site constant. La vitesse radiale, mesurée en fonction de l'azimut $\beta$ à chaque distance $r$, est fonction du vent horizontal $V_{h}$ (de direction $\beta_{0}$ ) et s'écrit :

$$
V_{r}=V_{h} \cos \alpha \cos \left(\beta-\beta_{0}\right)+V_{z} \sin \alpha
$$

Lorsque $V_{h}$ et $V_{z}$ sont suffisamment homogène et stationnaire dans l'aire explorée par le radar, on obtient alors une détermination des profils verticaux de $V_{h}$, $\beta_{0}$ (module et direction du vent horizontal) et $V_{z}$ en repérant, à chaque altitude, les maximum $\left(\beta=\beta_{0}\right)$, minimum $\left(\beta=\beta_{0}+\pi\right)$, la phase et la valeur moyenne de la courbe sinusoidale que dessine $V_{r}(\beta)$ à chaque tour d'antenne.

En pratique, l'hypothèse d'uniformité horizontale des composantes du vent est trop restrictive. Un perfectionnement important, dû à Browning et Wexler [12], consiste à écrire les composantes de $V_{n}$ sous forme d'un développement de Taylor limité aux dérivées premières et à effectuer une analyse de Fourier à l'ordre 2, du signal acquis au cours des divers VAD. On montre alors qu'à partir des coefficients de Fourier obtenus, on peut déterminer, en fonction de l'altitude, la vitesse, la direction, la divergence, la déformation et l'axe de dilatation du vent horizontal. Ces quantités, que l'on réfère à la verticale du radar, sont en fait représentatives à l'échelle de l'aire couverte par le balayage du faisceau, typiquement 10 à $50 \mathrm{~km}$ de rayon. La divergence du vent horizontal est fournie par l'expression:

$$
\operatorname{div} V_{h}=2\left(a_{0}-V_{T} \sin \alpha\right) /\left(r \cos ^{2} \alpha\right)
$$

où $a_{0}$ est 1'harmonique zéro du développement de Fourier de $V_{r}(\beta)$. Le profil de vitesse verticale $w(z)$ peut alors être obtenu en intégrant l'équation de continuité de la masse :

$$
\operatorname{div} V_{h}+(1 / \rho) \partial(\rho w) / \partial z=0
$$

à partir du sol (où $w=0$ ), $\rho$ étant la densité de l'air à l'altitude $z$. L'analyse doit être effectuée à site faible (typiquement $\alpha<10^{\circ}$ ) de façon à réduire la contribution du deuxième terme (dû à $V_{T}$ ) que l'on doit estimer dans l'équation (9).

Des améliorations ultérieures, consistant à traiter les données en volume, ont été apportées par plusieurs auteurs (Wald teufel et Corbin, [13]; Matejka et Srivastava [14]) et l'analyse permet alors de déterminer également le profil vertical de vitesse de chute $V_{T}(z)$ des précipitations sans que l'on soit obligé de confiner la technique à des sites d'exploration faibles au-dessus de 1'horizon. Un exemple d'une telle détermination par Matejka et Srivastava [14] est présentée dans la figure 3.a. L'accord obtenu entre le profil de vitesse de chute déduit de la technique VAD et celui mesuré directement par une visée verticale du radar, est remarquable. La transition abrupte de vitesse dans la région de transformation de la neige en pluie, à la traversée de l'isotherme $0^{\circ} \mathrm{C}$ (situé vers $3,5 \mathrm{~km}$ d'altitude) est parfaitement res-
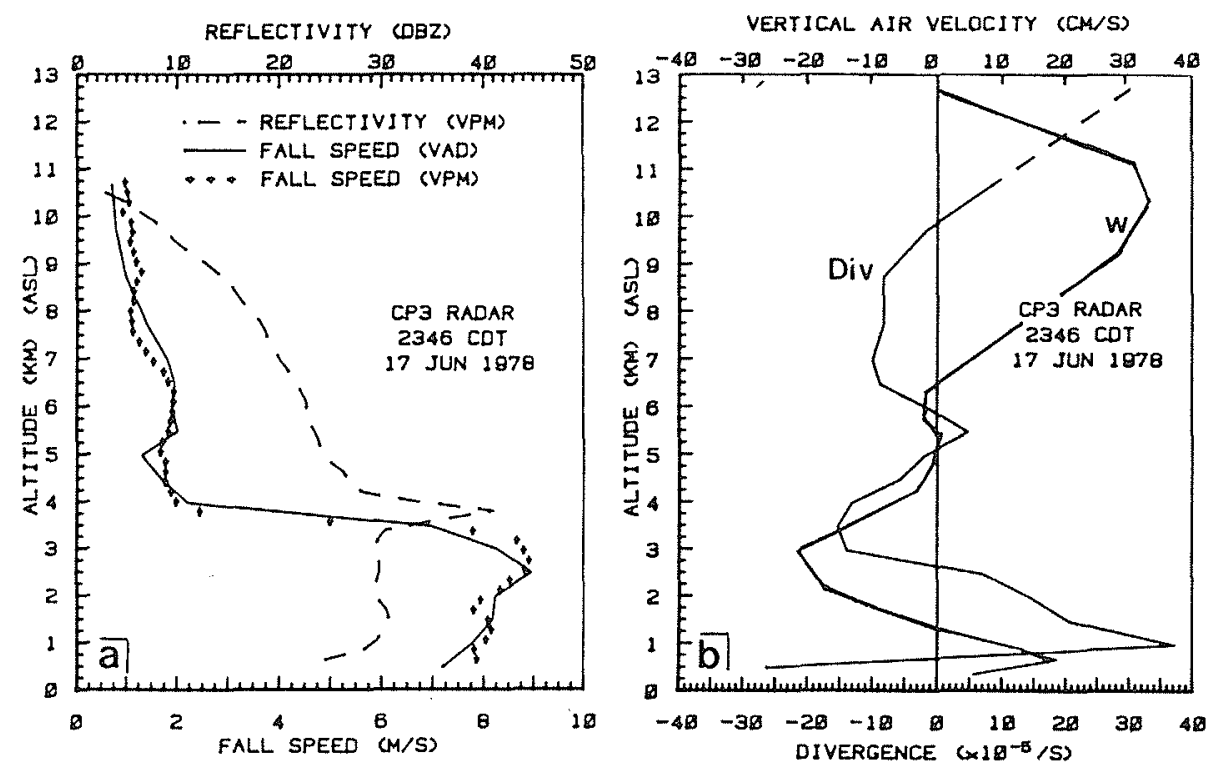

Figure 3 - Profils verticaux de paramètres de l'analyse VAD au sein d'une pluie stratiforme à l'arrière d'une ligne de grains dans le Nord-Ouest de I'llinois (USA) le 17 juin 1978. (a) Vitesse de chute des hydrométéores (VAD) comparée à celle déduite de mesures radar en tir vertical (VPM) auxquelles se rapportent également le profil de facteur de réflectivité. (b) Divergence de la vitesse horizontale de l'air et vitesse verticale de l'air déduite par intégration de la courbe précédente. D'après Matejka et Srivastava [14]. 
tituée et ceci témoigne de la qualité de l'analyse. La figure 3.b présente les profils verticaux obtenus pour div $V_{h}$ et pour la vitesse verticale de l'air, qui n'excède pas $40 \mathrm{~cm} \mathrm{~s}^{-1}$ mais s'exerce à l'échelle de l'air couverte par l'analyse, soit environ $5000 \mathrm{~km}^{2}$.

$\mathrm{La}$ principale limite de la technique VAD provient des défauts de linéarité du champ de vent au sein du système étudié. Toutefois, dans certains cas, les irrégularités non résolues par l'analyse, peuvent être interprétées en termes de mouvements ondulatoires (Testud et al. [15]) ou turbulents (Bryant et Browning [16]) d'échelle inférieure à celle de l'aire d'exploration. La méthode est donc bien adaptée à l'étude de la convection faible, étendue horizontalement et suffisamment homogène. C'est le cas des pluies stratiformes généralement associées aux systèmes frontaux dépressionnaires à moyenne latitude. Le type de résultat que l'on peut y atteindre, concernant la détermination de la structure de l'écoulement de l'air à moyenne échelle, est illustré dans la figure 4 (Wilson et al. [17]).

Dans ce contexte, il est intéressant de mentionner l'usage possible de cette technique pour l'étude du bilan de l'eau précipitante. En effet, muni du profil en altitude de $w$ et connaissant (à l'aide de sondages météorologiques) le rapport de mélange saturant $m$ (par rapport à l'eau ou la glace, le cas échéant), on peut calculer l'intégrale verticale du taux de condensation dans une colonne d'air supposée saturée, au-dessus du radar et déterminer ainsi un taux de précipitation moyen théorique $R_{t}$ donné par :

$$
R_{t}=\int_{z_{1}}^{z_{2}} \rho(z) w(z)(d m / d z) d z
$$

$z_{1}$ et $z_{2}$ sont les altitudes de base et de sommet du nuage. La comparaison entre $R_{t}$ taux précipitant que l'on observerait si toute l'eau condensée était précipitée, et le taux $R$ réellement observé au sol, constitue une mesure de l'efficacité $\epsilon=R / R_{t}$ du système à convertir l'eau nuageuse en précipitations. Dans l'exemple de la figure 4, est également indiquée une comparaison entre le taux $R_{t}$ déduit de l'analyse $\mathrm{VAD}$, celui observé au sol à l'aide d'un pluviomètre situé au pied du radar et celui déduit de mesures de réflectivité radar en tir vertical. Dans le cas présent, où $R_{t}$ est représentatif d'une aire de $20 \mathrm{~km}$ de rayon environ autour du radar, l'efficacité $\epsilon$ de la précipitation est estimée à $83 \%$, durant les 6 heures
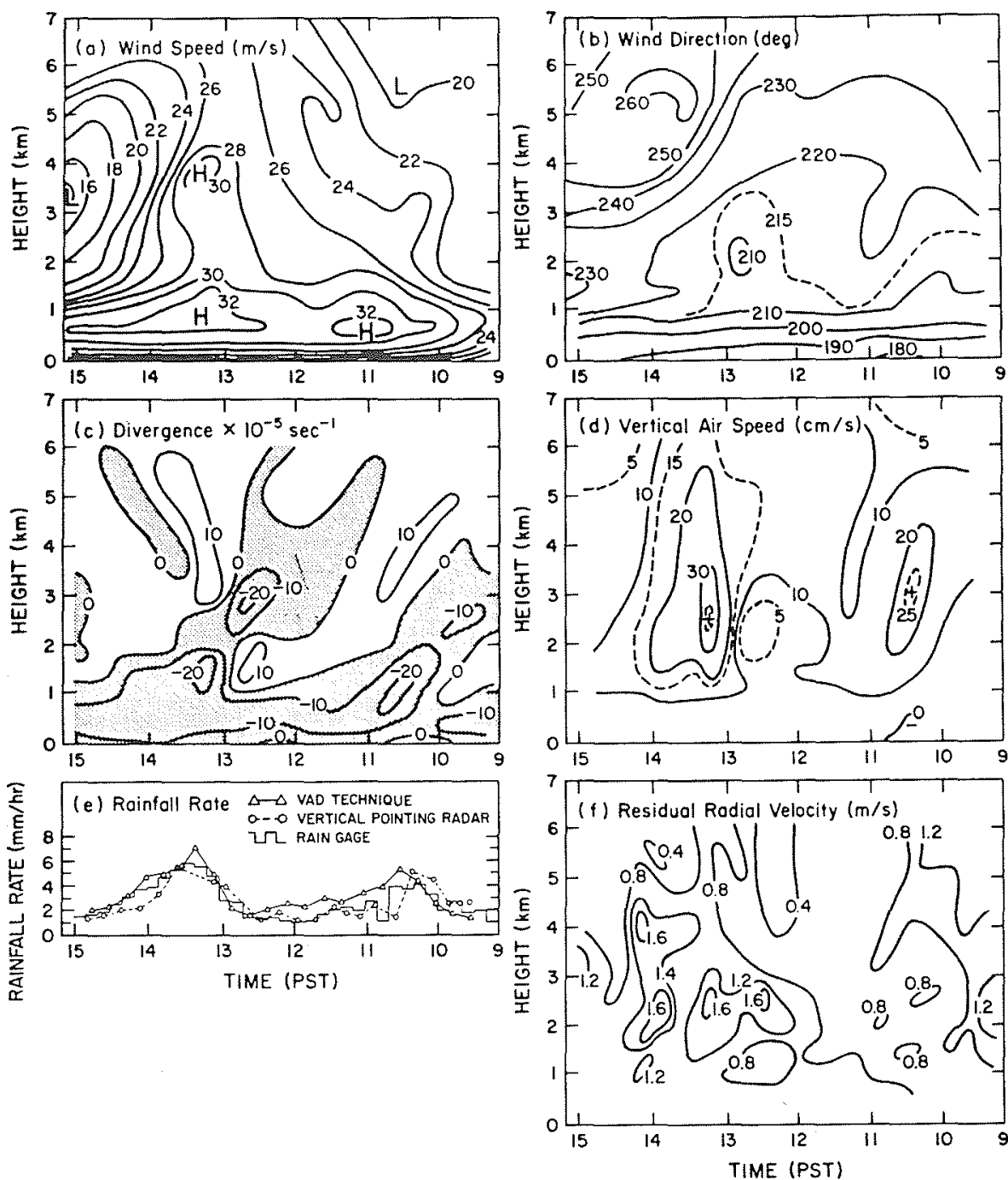

Figure 4 - Caractéristiques à moyenne échelle du vent et des précipitations au sol, déduites de la méthodologie VAD durant 6 h d'obser vation d'une pluie stratiforme associée à un front occlus, le 4 mai 1979 à Ocean Shores, Washington (USA). D'après Wilson et al. [17]. 
d'observation. L'étude de différents types de bandes de pluies frontales observées dans le nord-ouest des Etats-Unis (Hobbs et al. [18], Wilson et al. [19]) révèle une grande disparité des valeurs de $\epsilon$, allant de $90 \%$ à moins de $50 \%$. Dans ce dernier cas, l'inefficacité des processus de conversion "eau nuageuse/précipitations" laisse subsister une grande quantité d'eau en suspension dans le nuage.

L'étude des causes de telles différences et des moyens éventuels de les réduire constitue un sujet de recherche prometteur.

\subsection{Réseau de radars Doppler : restitution du champ de vitesse tridimensionnel.}

Dans l'étude de la convection précipitante développée (orages) l'hypothèse de relative uniformité du champ de vent ne s'applique plus. Lorsqu'on dispose de plusieurs radars Doppler opérant simultanément au sein d'un même système, chaque vitesse radiale $V_{i}(x, y, z)$ mesurée en un point de l'espace est une fonction des trois composantes $u, v, w$ du vent et de la vitesse de chute $V_{T}$ de la précipitation. On sait en général estimer cette dernière par une relation du type $V_{T}-Z$ et la combinaison de trois mesures de vitesse $V_{i}(i=1,2,3)$ non colinéaires à partir de trois radars donne donc, en principe, accès à la détermination des trois composantes du vent.

En réalité, la configuration d'un réseau est généralement adaptée à l'observation de systèmes convectifs à sites faibles, loin des radars (l'espacement typique est de l'ordre de 30 à $50 \mathrm{~km}$ ) de façon à disposer d'une large zone d'observation possible avec une différentiation géométrique suffisante entre les directions de visée des divers instruments en chaque point de l'espace.

Dans ces conditions, toutes les études indiquent que, quel que soit le nombre de radars mis en cuvre, on peut obtenir une restitution précise du vent horizontal et de sa divergence mais le calcul de la vitesse verticale nécessite toujours l'intégration de l'équation de continuité de la masse. On conçoit alors que l'utilisation de deux radars suffisent pour résoudre le problème. Cette procédure, dénommée COPLAN, la première proposée historiquement (Lhermitte et Miller [20]), prévoit une exploration de l'espace par les deux radars, par plans successifs (balayés quasi-simultanément) passant par la ligne de base des radars et dont on fait varier progressivement l'inclinaison par rapport au plan horizontal, de façon à réaliser une exploration en volume du système précipitant.

Dans chaque plan, on peut alors reconstituer deux composantes orthogonales de la vitesse du vent suivant un maillage cartésien régulier. La troisième composante, orthogonale aux maillages des divers plans, est obtenue par intégration de l'équation de continuité de la masse. Ceci revient en fait à travailler en coordonnées cylindriques, l'axe du cylindre étant la ligne de base des radars. Des procédures d'interpolation permettent ensuite de reconstituer le champ de vitesse tridimensionnel $(u, v, w)$ dans un maillage cartésien conventionnel suivant des axes $x, y, z$ où $z$ est la verticale. Le traitement des données de plusieurs radars procède d'une approche similaire. Toutefois, les balayages de l'espace par les divers instruments sont généralement effectués de façon indépendante et la reconstitution du champ de vent s'effectue directement dans un maillage cartésien conventionnel $x, y, z$.

Avec les performances des systèmes actuels, il est possible d'obtenir une image de la dynamique tridimensionnelle de l'air dans un système convectif occupant un volume de l'ordre de $50 \times 50 \mathrm{~km}^{2}$ (horizontalement) $x 15 \mathrm{~km}$ (verticalement) et sur un maillage cartésien de l'ordre de $1 \mathrm{~km}$ horizontalement et $500 \mathrm{~m}$ verticalement, toutes les $5 \mathrm{mn}$ environ. Les travaux fondamentaux accomplis pour certifier la qualité des mesures obtenues ont surtout porté sur les techniques de filtrage des mouvements turbulents (d'échelle inférieure à la maille de restitution), sur les possibilités de corriger objectivement les effets du déplacement et de l'évolution propre du système au cours de l'échantillonnage, sur les procédures d'intégration de l'équation de continuité de la masse et enfin sur l'optimisation de la configuration géométrique des réseaux, pour minimiser les erreurs (Doviak et al. [21]; Ray et al. [22] ; Testud [23]).

Pour illustrer la méthodologie décrite, on présente un exemple de résultats obtenus avec le système RONSARD dans une ligne de grains tropicale observée en Côte d'Ivoire durant l'expérience COPT 81 . La figure 5 représente le champ de réflectivité à basse altitude, déduit des mesures de l'un des radars à 04.53 TU. Le système est de grande dimension, se déplace vers le sud-ouest à $20 \mathrm{~m} \mathrm{~s}^{-1}$ en conservant sa structure générale et son intensité pendant de longues heures. Il est formé d'un violent front convectif à l'avant, auquel sont associées

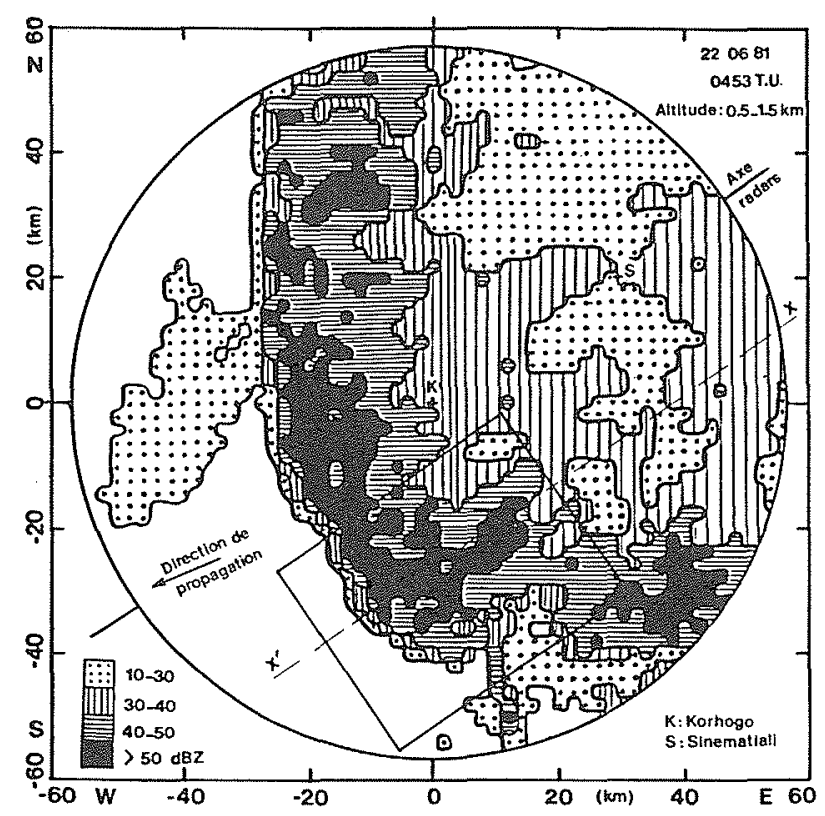

Figure 5-Structure horizontale đu facteur de réflectivité observé à partir du radar KORHOGO (K) à 04.53 TU dans la ligne de grains du 22 juin 1981, au cours de l'expérience "COPT 81 " dans le Nord de la Côte d'Ivoire. Les fortes précipitations $(>50 \mathrm{~mm} / \mathrm{h}$ ) dans le front convectif à l'avant du système sont suivies d'une large zone de pluie stratiforme (quelques $\mathrm{mm} / \mathrm{h}$ ). La région encadrée est celle où a été restitué le champ de vitesse tridimensionnel à partir des mesures des deux radars ( $K$ et $S$ ) Doppler du système RONSARD à 04.18 TU. D'après Chong (communication privée). 

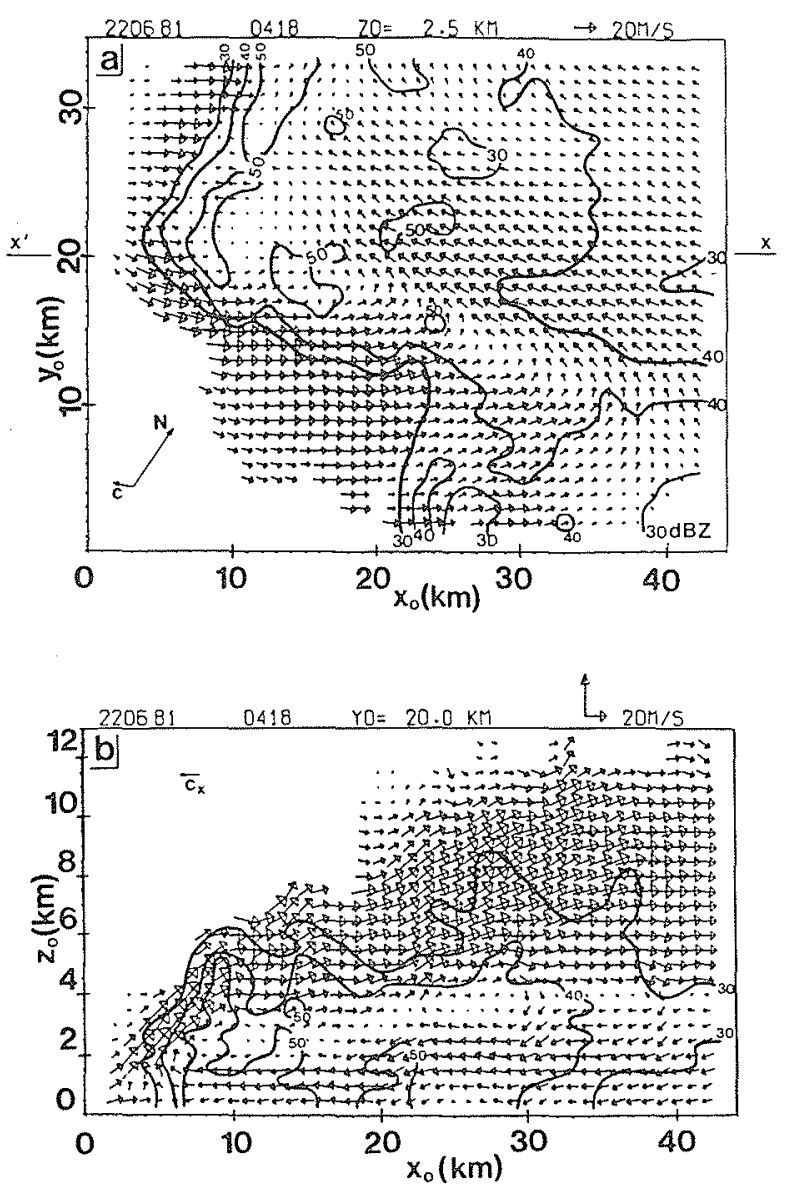

Figure 6 - (a) Champ de vitesse horizontal (altitude $2.5 \mathrm{~km}$ ) reconstitué à $04.18 \mathrm{TU}$ dans la région de la ligne de grains encadrée sur la figure 5 et isocontours de facteur de réflectivité. (b) Ecoulement de l'air et isocontours de facteur de réflectivité dans le plan vertical de la coupe $\mathrm{X}^{\prime} \mathrm{X}$ indiquée sur les figures 5 et $6 a$ dans les deux cas, il s'agit de la vitesse de l'air dans le referentiel lié au système en déplacement à la vitesse $C$ (ou $\mathrm{C}_{\mathrm{x}}$ ). D'après Chong (communication privée).

des pluies très intenses (ligne d'orages), suivi d'une traîne de précipitations stratiformes s'étendant jusqu'à $200 \mathrm{~km}$ vers l'arrière. Sur la figure est également indiquée la région où, en tenant compte du déplacement du système, a été obtenue l'une des reconstitutions du champ de vent tridimensionnel à l'aide de la méthodologie COPLAN. La figure 6.a représente le champ de vitesse horizontal, à $2.5 \mathrm{~km}$ d'altitude, obtenu à $04.18 \mathrm{TU}$ dans cette région. La figure $6 . \mathrm{b}$ indique la stucture de l'écoulement de l'air dans une coupe verti. cale suivant un axe proche de la direction d'advection. Dans les deux cas, il s'agit du champ de vent relatif dans le système en mouvement, déduction faite de la vitesse d'advection d'ensemble.

Sans détailler l'analyse de ces résultats, ou d'autres similaires acquis au cours des quatre heures d'observation de l'évènement, on résume ci-dessous l'interprétation qu'ils ont permis d'élaborer (Roux et al. [24]). Dans la partie la plus active du front de convection, l'écoulement de l'air est quasi-bidimensionnel (Fig. 6.b). l'ascendance d'air chaud à l'avant, résulte de la poussée d'une masse d'air froid, injectée par l'arrière dans les deux premiers kilomètres d'altitude. Cette masse d'air froid joue un rôle déterminant dans le déplacement et l'entretien du front convectif. Les pluies violentes du front convectif sont formées essentiellement en phase liquide car elles se situent dans une région où l'extension verticale de l'écoulement n'excède guère le niveau de glaciation (isotherme $0^{\circ} \mathrm{C}$ vers $4.2 \mathrm{~km}$ ). Le refroidissement de la masse d'air à l'arrière est produit et entretenu par l'évaporation partielle des précipitations dans l'air non saturé; ce dernier subissant une descendance globale à moyenne échelle, bien identifiée à partir des résultats de l'analyse $\mathrm{VAD}$, obtenus régulièrement dans la région de pluies stratiformes. Ce schéma explicite de fonctionnement dynamique de la convection précipitante dans une ligne de grains tropicale devra être confirmé par des études quantitatives en cours de développement.

Le traitement complet des données d'un réseau de radars Doppler pour reconstituer le champ de vitesse tridimensionnel (et le champ de réflectivité associé) nécessite l'utilisation de programmes lourds sur des ordinateurs performants. Néanmoins, l'obtention de la dynamique tridimensionnelle d'un système convectif, dans un domaine et avec une résolution comparable à ceux des modèles théoriques, constitue un enjeu suffisant pour justifier l'ampleur des travaux réalisés depuis une dizaine d'années. En effet, en aval des descriptions des champs de vitesse et de précipitations, apparaît le développement des études visant à comprendre et modéliser les processus dynamiques et thermodynamiques qui régissent la convection profonde et les mécanismes de production et d'entretien des précipitations.

\section{Conclusion et perspectives.}

Dans l'étude des systèmes précipitants, le radar Doppler est encore aujourd'hui essentiellement un instrument de recherche. On ne conçoit plus d'expérience importante dans ce domaine sans le déploiement d'un réseau de tels radars, assorti de moyens de mesures météorologiques conventionnels et éventuellement d'avions instrumentés. Les méthodologies classiques que nous avons présentées, sont régulièrement utilisées en fonction des caractéristiques des évènements étudiés et permettent d'accomplir des progrès décisifs dans l'étude fondamentale de la physique des précipitations stratiformes ou orageuses.

Toutefois, le besoin de développer des applications opérationnelles n'a pas échappé aux expérimentateurs (Wilson et al. [17] [25]). Il s'agit surtout ici de contribuer aux besoins de prévision à courte échéance (quelques heures). L'utilisation du radar Doppler repose alors sur l'obtention rapide d'images de réflectivité et de vitesse radiale numérisées et. visualisées sur écran couleur, qui permettent de reconnaître les signatures de phénomènes caractéristiques, sans nécessiter l'appication des lourds traitements élaborés pour les besoins de recherche fondamentale. A titre d'exemple, l'analyse des images permet de localiser avec précision les zones frontales, de prévoir leur déplacement à échéance de quelques heures. L'estimation de la hauteur d'eau précipitable à partir de la méthodologie $\mathrm{VAD}$ (voir 
section 3.1) dans les précipitations étendues, peut faire l'objet d'une intégration en temps réel, avec un intérêt évident pour les besoins de l'hydrologie ou de l'agriculture. Dans l'étude des orages sévères, les images de radar Doppler fournissent des signatures caractéristiques des tornades, des fronts de rafale, des descendances violentes, des cisaillements de vent intenses. Il devient ainsi possible de prévoir l'intensité et la trajectoire de tels phénomènes dangereux (par exemple près des aéroports) et éventuellement générateurs de dégats matériels. Ces considérations ont conduit certains pays à envisager, dans l'avenir, le déploiement d'un réseau de radars météorologiques Doppler. Aux Etats-Unis, le projet NEXRAD prévoit le remplacement des radars conventionnels du réseau (environ 160) par des systèmes Doppler (Bonewitz [26]).

Enfin, il convient de mentionner rapidement deux développements prospectifs prévus pour la prochaine décennie (Serafin et al. [27]). Le premier, dont la faisabilité technique est démontrée, concerne la réalisation d'un radar Doppler à balayage très rapide. Un tel système, à l'étude aux Etats-Unis, devrait balayer 1'hémisphère environnant en $30 \mathrm{~s}$ environ et permettre ainsi de s'affanchir de façon spectaculaire, des limites inhérentes aux analyses actuelles qui mettent en jeu des temps d'observation environ dix fois plus élevés. Le deuxième développement concerne la possibilité d'embarquer un radar Doppler sur avion, dont la mobilité devrait permettre de réaliser des cartographies à haute résolution des orages, de détecter l'intensité de foyers orageux en développement (mesure de la vitesse verticale) et des cyclones tropicaux, en particulier sur l'océan. Malgré des essais expérimentaux prometteurs, la rentabilité d'un tel système n'est pas encore admise, compte-tenu de son coût très élevé.

En conclusion, il est clair que l'utilité du radar Doppler dans l'étude de la physique des précipitations n'est pas en question. Outils de recherche et d'application, on peut prévoir une intensification permanente de leur utilisation au cours des années à venir. Dans ce contexte, on remarquera que la France est un des pays les mieux équipés après les Etats-Unis. Elle possède en effet trois systèmes (dont un double), très complémentaires, destinés surtout à la recherche. Souhaitons que les moyens nécessaires à leur exploitation scientifique restent en rapport avec l'ampleur des taches à accomplir.

\section{Références}

[1] BATTAN L.J. - Radar observation of the atmosphere. The University of Chicago Press., 324 pp. (1973).

[2] SAUVAGEOT H.-Radarmétéorologie, Télédétection active de l'atmosphère. Ed. Eyrolles, Paris, 296 pp. (1982).

[3] BATTAN L.J. - Some observations of vertical velocities and precipitation sizes in a thunderstorm.J. Appl. Meteor., 3, 415-420 (1964).

14] BATTAN L.J. and THEISS J.B. - Measurements of vertical velocities in convective clouds by means of pulsed-Doppler radar. J. Atmos. Sci., 27, 293-298 (1970).

[5] MARSHALL J.S and PALMER W.M.K. - The distribution of raindrops with size. J. Meteor., 5, 165-166 (1948).

[6] ROGERS R.R. - An extension of the $Z-R$ relationship for Doppler radar. Prep. 11th Wea. Radar Conf., Amer. Meteor. Soc., Boston, 158-160 (1964).

[7] HAUSER D. and AMAYENC P. - A new method for deducing hydrometeor-size distributions and vertical air motions from Doppler radar measurements at vertical incidence. J. Appl. Meteor., 20, 547-555 (1981).

[8] ATLAS D., SRIVASTAVA R.C. and SEKHON R.S. Doppler radar characteristics of precipitations at vertical incidence. Rev. of Geoph. and Space Physics, 11, 1, 1-35 (1973).

19] NUTTEN B., AMAYENC P., CHONG M., HAUSER D., ROUX F. and TESTUD J. - The Ronsard radars: $A$ versatile $C$ dual Doppler facility. IEEE Trans. Geosci. Electron. GE-17, 281-288 (1979).

[10] HAUSER D. and AMAYENC P. - Drop-size distribution and vertical air motions in a thunderstorm as inferred from Doppler radar observations at vertical incidence. $J$. Rech. Atmos., 14, 439-455 (1980).

[11] LHERMITTE R.M, and ATLAS D. - Precipitation motion by pulse Doppler radar. Prep. $96 \mathrm{~h}$ Wea. Radar Conf., Amer. Meteor. Soc., Boston, 218-223 (1961).

[12] BROWNING K.A. and WEXLER R. - A determination of kinematic properties of a wind field using Doppler radar. J. Appl. Meteor., 7, 105-113 (1968).
[13] WALDTEUFEL P. and CORBIN H. - On the analysis of single - Doppler radar data. J. Appl. Meteor., $18532-542$ (1979).

[14] MATEJKA T.J. and SRIVASTAVA R.C. - Doppler radar study of a region of widespread precipitation trailing a Midlatitude squall line. Prep. 20th Conf. on Radar Meteorology, Amer. Meteor. Soc., Boston, 353-357 (1981).

[15] TESTUD J., BREGER G., AMAYENC P., CHONG M., NUTTEN B, and SAUVAGET A. - A doppler radar observation of a cold front - three-dimensional air ciculation, related precipitation system and associated wavelike motions. J. Atmos. Sci., 37, 78-98 (1980),

[16] BRYANT G.W. and BROWNING K.A. - Multi-level measurements of turbulence over the sea during the passage of a frontal zone. Quart. J. Roy. Meteor Soc., 101, 35-54 (1975).

[17] WILSON J., CARBONE R.E. and McCARTHY J, - Forecasting applications of Doppler radar. Athmospheric Technology (NCAR, Boulder), 13, 105-118 (1981).

[18] HOBBS P.V., MATEJKA T.J., HERZEGH P.H., LOCA TELLI J.P. and HOUZE R.A. Jr. - The mesoscale and microscale structure and organization of clouds and precipitation in mid-latitudes cyclones. I : A case study of a cold front. J. Atmos. Sci. 37, 568-596 (1980).

[19] WILSON J, CARBONE R.E., and RAMSAY B. - Precipitation and precipitation efficiencies derived from single Doppler radar. Prep. 20th Conf. on Radar Meteorology, Amer. Meteor. Soc., Boston, 461-468 (1981).

[20] LHERMITTE R.M. and MLLLER L.J. Doppler radar methodology for the observation of convective storms. Prep. 14th Radar Meteor. Conf., Amer. Meteor. Conf., Amer. Meteor. Soc., Boston, 133-138 (1970).

[21] DOVIAK R.J., RAY P.S., STRAUCH R.G. and MILLER L.J. - Error estimation in wind fields derived from dualDoppler radar measurement. J. Appl. Meteor., 15, 868878 (1976). 
[22] RAY P.S.,ZIEGLER C.L.,BUMGARNER W. and SERAFIN R.J. - Single and multiple Doppler radar observations of tornadic storms. Month. Weath. Rev., 108, 1607-1625 (1980).

[23] TESTUD J. - These dimensional wind field analysis from radar data. Cours de l'Ecole d'Eté NATO/ASI, Bonas (France), dans NATO/ASI on Mesoscale Meteorology observations. Theories and Models, D. REIDEL Publ. Company (1982).

[24] ROUX F., TESTUD J., PINTY B. and CHALON J.P. Three dimensional motions within an African squall line observed during "COPT 81 " experiment. Prep. of Conf. on Cloud Physics, Amer. Meteor. Soc., Boston, 547-550 (1982).

[25] WILSON R., CARBONE R., BAYNTON H, and SERAFIN R. - Operational application of Meteorological Doppler radar. Bull. of Amer. Meteor. Soc., 61, (10), 1154-1168 (1980).

[26] BONEWITZ J.D. - The NEXRAD program - an overview. Prep. 20th Conf. on Radar Meteorology. Amer. Meteor. Soc., Boston, 757-761 (1981).

[27] SERAFIN R., LHERMITTE R. and ATLAS D. - Radar meteorology - Developments in the 1980's. Atmospheric Technology (NCAR, Boulder), 13, 135-143 (1981).

\section{Discussion}

Sur l'intervention de M. PIRCHER concernant la prise en compte des nuages dans le bilan de l'eau précipitante, $M$. $A M A Y E N C$ explique que l'on suppose que le milieu reste saturé. De ce fait, aucun terme ne représente l'évaporation des précipitations, alors que cette quantité serait à considérer si l'on se plaçait en milieu non saturé. Il remarque que malgré cette approche simplifiée, le bilan reste équilibré (à $12 \%$ près), mais qu'un modèle plus complet pourrait être adopté.

M. AMAYENC indique à la demande de M. PARSY que la longueur d'onde du système Ronsard est de $5,3 \mathrm{~cm}$.

M. SAUVAGEOT remarque que sur les exemples présentés, le niveau de condensation s'étend de $100 \mathrm{~m}$ dans la partie convective à $4000 \mathrm{~m}$ dans la partie stratiforme.
M. AMAYENC précise que le calcul nécessite surtout la connaissance de la zone où la composante verticale de la vitesse s'annule, ce qui ne correspond pas forcément au niveau de condensation.

En conclusion, M. LABROUSSE constate que cette première séance constitue une bonne évocation de l'état de la technique radar, actuelle et future, complétée par la présentation d'un premier exemple. Il indique que la Météorologie Nationale considère cette approche comme la plus prometteuse dans le domaine de la mesure des précipitations et de la prévision à court terme, et que les projets entrepris dans ce domaine seront détaillés dans les séances ultérieures.

M. LABROUSSE, pour terminer, remercie tous les participants.

\section{Abstract}

\section{The role of the Doppler radar in the study of precipitation}

Over the last ten years the Doppler meteorological radar has become a popular instrument for studying precipitation. Even today it still remains principally a research instrument for studying precipitation systems although development work on possible operational applications was recently started.

In the introduction the operating principle of the Doppler radar is recalled showing that, in addition to the power of the echo received as in the case of conventional radar, it also can measure the radial velocity of cones in each field observed.

The three conventional methods of using the Doppler radar for scientific purposes are then obtained. For each method, the principle together with recent modifications is described and physical quantities obtainable are illustrated by significant experimental results. The methodology of vertical shooting (part II) is examined whereby data on the vertical air speed and granulation of precipitation at the radar zenith can be recorded.Part III describes the conical speed method with one radar and the use of networks with two or more radars. The latter make it possible to observe the three-dimensional dynamics of air movement within a given precipitation system.

Part IV briefly indicates recent operational applications based on rapid recognition of typical signals in reflectivity and radial velocity pictures which are provided with numbers and displayed on a colour screen. They are basically used for short-term forecasting of violent and dangerous phenomena (severity of near thunderstorms, tornadoes, gust fronts, turbulent zones...). Two possible future developments expected over the next few years are also mentioned: the rapid sweep Doppler radar and the airborne Doppler radar. 